\title{
Study of Group Route Optimization for IoT enabled Urban Transportation Network
}

\author{
Koh Song Sang \\ Department of Computer Science, \\ Liverpool John Moores University, \\ Liverpool, L3 3AF, UK \\ S.S.Koh@2014.ljmu.ac.uk \\ Po Yang \\ Department of Computer Science, \\ Liverpool John Moores University, \\ Liverpool, L3 3AF, UK \\ B.Yang@ljmu.ac.uk
}

\author{
Bo Zhou \\ Department of Computer Science, \\ Liverpool John Moores University, \\ Liverpool, L3 3AF, UK \\ S.S.Koh@2014.ljmu.ac.uk \\ Zaili Yang \\ Department of Computer Science, \\ Liverpool John Moores University, \\ Liverpool, L3 3AF, UK \\ Z.Yang@ljmu.ac.uk
}

\begin{abstract}
Traffic congestion is always a major issue in urban planning, especially when the vehicles in the roadway keep growing and the local authorities are lack of solutions to manage or distribute the traffics in the city. Although there are several factors that may cause traffic congestion, inefficiency in traffic management is always the main issue. Additionally, the most traditional methods of resolving traffic congestion or rerouting algorithm are mainly designed for individuals' benefits, by simply planning a driver's route based on minimum travel time or shortest path accordingly. There is lack of consideration in group benefit or urban development. However, with the development of technologies in Internet of Things (IoT), vehicle to vehicle (V2V) or Vehicle to Infrastructure (V2I) communications, group based routing becomes achievable. Instead of optimizing the routing path for individual drivers, this paper studies how to develop a new method to provide new routing method based on vehicles' similarities in a specific urban's transportation environment.
\end{abstract}

Index Terms - Traffic Congestion, Urban Development, Transportation Network, Route Optimization

\section{INTRODUCTION}

Traffic congestion is always a major issue in urban planning, especially when the vehicles on the roadway keep growing and the urban infrastructure is not upgraded in equal speed to accommodate the growing number of vehicles. Besides, although many different methods about route optimization had been widely studied, most of them are only designed for individual users and lack of consideration from the urban wise in terms of sustainability and resilience. Furthermore, local authorities to date still lack efficient technical solutions to manage or distribute the traffics in the urban transportation network. To fulfill such research gaps, it is essential and significant to investigate the state of the art of research on managing the urban transportation networks, and a new routing algorithm needs to be designed for urban perspective.

With the wireless sensor networks technologies are getting more mature and reliable, these become a main factor for the development of IoT in smart transportation. For instance, the mobility of sensor nodes enables the Vehicle to Vehicle (V2V),
Vehicle to Roadside (V2R) or Vehicle to Infrastructure (V2I) communication, which means the data sharing and collection are more reliable and fast between vehicles, roadside and infrastructures. By accessing the realtime traffic data, the vehicle traffic management system controller could response instantly to control the urban traffic flow [10].

Route optimization is a general method for resolving Vehicle Routing Problem (VRP) which is described as the problem of designing optimal delivery or collection routes from one or several depots to a number of geographically scattered cities or customers, subject to side constraints [15].

Traditionally, most of the past routing algorithms are based on individual benefit, such as finding the shortest path, or minimizing the travel time over the traffic. There is a lack of consideration in urban wise and the correlation of different vehicle types and driving behaviors. Therefore, this project focuses on evaluating a "group" routing algorithm based on vehicle or driver similarities. And also instead of aiming to minimize the individual driver's travel time, this project aims to reduce the "average" travel time of every vehicle in specific urban transportation network by approaching the group route algorithm. This believingly could bring the group benefit for every driver in urban transportation network.

Therefore, the routing algorithm firstly needs to categorize the vehicles based on their characteristic, for instance vehicle type or driving behavior. Then giving the routing instruction to each vehicle accordingly based on their destination and traffic situation. This approach will then be compared with standard routing approach to determine its benefits.

Additionally, since urban transportation network should give emergency unit a special treatment while managing the urban traffics, this project also plans to work on an extra strategy for emergency unit, which will be able to give them the routing instruction by breaking some fundamental rules, such as drive through the traffic light, or drive in the opposite direction lane 
to make sure they could arrive their destination as soon as possible.

The remaining of the report is as follows: section 2 provides a systematic review on the existing research on traffic management and routing algorithm. Section 3 shows the general idea of group route optimization. Section 4 discusses the prototype, section 5 describe a simple experiment of group route optimization and lastly section 6 gives the conclusion and future plan of the research.

\section{RELATED WORK}

Vehicle routing problem is major challenge in management of transport network. Route optimization is a general method for resolving Vehicle Routing Problem (VRP) which is described as the problem of designing optimal delivery or collection routes from one or several depots to several geographically scattered cities or customers, subject to side constraints [1].

Dijkstra [2] proposed a static algorithm to find the shortest path without considering any external factor such as congestion, accident, or average vehicle speed. However, this method is not practical enough as the traffic of transport network is changing over time and has specific constraints. Therefore, vehicle routing optimization should always optimize the path continuously with the real-time information and adapt to the latest circumstance.

Ant-colony algorithm was first proposed by Dorigo et al. in paper [3] which was inspired by the natural behaviour performed by ants in finding food resources. In this natural phenomenon, ants leave pheromone trails to allow other ants to track the path and eventually find the food resources. Previous experiments have proven that ants can find the shortest route between two individual sections. Therefore, ant-colony algorithm is widely applied to solve vehicle routing problem, although some modifications have been applied depending on different circumstances.

Nahar and Hashim [4] proposed a traffic congestion control method based on different preferences to create an optimal traffic system. Figure 3 shows the method used in determining the optimum path. These preferences allow the algorithm to reduce average travelling time by adjusting the ant colony variables. Their results show that the number of ants is directly correlated with the algorithm performance. Therefore, this method does not perform well when there is only a small number of agents in the network.

Kammoun et al. [5] however proposed an adaptive vehicle guidance system which can search for the best path in a smarter way by using real-time changes in the network. To achieve dynamic traffic control and improve driver's request management, this method used three types of agents, which are:

- Intelligent vehicle-ant agent (IVAA): standing for a vehicle and encapsulates a deliberative module for the selection of the best itinerary alternative.

- $\quad$ Road Supervisor Agent (RSA): Representing a software agent implanted in the server.
- $\quad$ City Agent (CA): Representing a software agent to manage the road network in the city to obtain a better exploitation of the network.

This method is focused on individual drivers and lack of consideration for the bigger picture of urban transportation network. It is difficult to manage a large, massive and complex urban transportation network.

Besides, a multi-agent evacuation model was introduced by Zong et al. [6] to minimise the total evacuation time for vehicles and balance the traffic load. Ants belong to one colony find their routes with the same properties. During the process of searching for routes, the two ant colonies will interact and share information with each other. Experiments have shown that Multi-Ant colony system is more effective than a single agent system.

Cong et al. [7] developed a model to optimise dynamic traffic routing by using a two-steps approach: network pruning and network flow optimisation. In the network-pruning phase, ant pheromone is removed after the best route is found by the agents to increase exploration rate. In the flow optimisation phase, which is based on Ant-Colony Optimization with the stench pheromone and colored pheromone, the agents correspond to the links selected in the network-pruning phase only. Moreover, this two-steps approach effectively reduces the computing burden when addressing complex and dynamic traffic control problems.

Kponyo et al. [8] proposed a distributed intelligent traffic system which uses vehicle average speed as a parameter to determine the traffic condition. This system guides vehicles to paths with low traffic. Therefore, this system selects the best path more efficiently comparing with scenarios where the agents select their path randomly.

For IoT enabled approaches on optimization for urban transportation network, Chao et al. [12] proposed an intelligent traffic management system which focuses on the use of radio frequency identification (RFID) as a form of traffic flow detection. The proposed intelligent traffic light control system (ITLCS) uses an RFID system to detect the number of seconds spent by vehicles on main roads and on side roads passing through detectors during a green light period and then transmits the detected data to the program system on chip (PSoC) microcontroller. They used Zig Bee wireless modules (a wireless network protocol for high-level communication used) to send real time data regarding weather conditions, air quality, and the detected vehicle registration information to the regional control centre. The proposed system performed remote transmission and reduce the risk of accident.

Bruno et al. [13] proposed two algorithms for collecting data which called GREEDY and Probabilistic Data Collection (PDC) for vehicular multimedia sensor networks. The project run the simulation on the proposed algorithm by using a VANETMobiSim and NS-2 simulator which showed GREEDY approach achieve more coverage area with less bandwidth.

Although most of the previous approaches provide certain optimization for urban transportation network, they are only considering individual benefit. For urban's sustainability and 
resilience wise, a modern traffic management approach need to be based on city perspective.

\section{GROUP ROUTE OPTIMIZATION}

The goal of this research is to propose and evaluate a group route algorithm to distribute the urban traffic based on group of vehicles that have similar characteristics. To this end, a general process flow for this approach is designed (Figure 1).

In the beginning, we assume that with the vehicle to vehicle (V2V) or vehicle to infrastructure (V2I) technologies, local authorities are able to collect all the required input to this approach, including traffic data (destination of each vehicle), vehicle type (size, weight, and priority), driving behaviour (aggressive, careful) and roadside condition (Wet level, construction).

With the input data, where urban traffic reaches "dangerous" level, local authorities, i.e. the central management system, could perform grouping approach in order to categorize the vehicles based on their similarities. After that it will execute the decision-making approach to re-route the vehicles to avoid the congestion. Besides, it will also give the top priority routing instruction for emergency units by allowing some exceptions to traffic rules when accident happens. For instance, allow the emergency units to drive through intersections when traffic lights are red, or drive in the opposite lane if necessary.

The local authorities will keep monitoring the urban traffic until the traffic situation back to normal level. If another unexpected event or potential congestion is detected, central management system will then approach the method again.

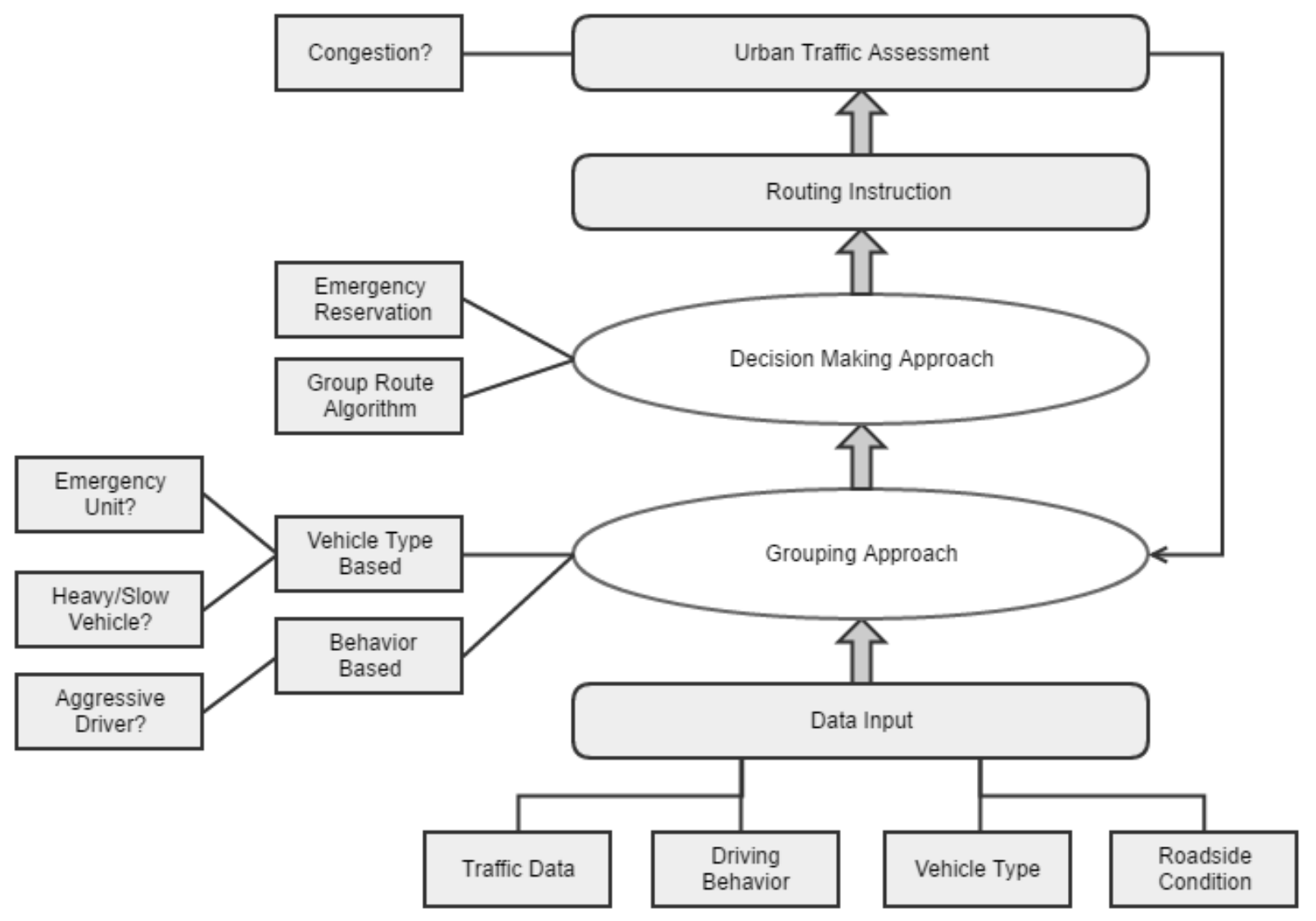

Figure 1 Process flow of the group routing approach

\section{REINFORCEMENT LEARNING}

Urban traffic distribution is fundamentally a problem of multicriteria decision making. The framework of reinforcement learning, in which an agent learns from model with optimal policy based on its environment, would be a good method to work on. Each action that the agent would take will lead to a reward or punishment with the new observation of the state. Through its learning progress, the agent will learn a distributed routing policy that could maximize the capacity of urban. This process could be treated as a Markov Decision Process (MDP), which ultimately aim for the best solution by optimizing specific policy step by step.Markov Decision Process contains 5 key elements as shown below [9]:

S: The collection of the states which could reflect the situation with specific properties.

A: A set of actions which could change the status. 
$\mathrm{P}_{\mathrm{SA}}$ : The probability of the state $\mathrm{S}$ change under action $\mathrm{A}$.

$\gamma$ : The discount factor.

$\mathrm{R}_{\mathrm{SA}}$ : The expect reward that state $\mathrm{S}$ change under action $\mathrm{A}$.

Considering urban transport network is rather a rapid and complex system (decision tree is hard to evaluate, and multiple decisions need to be made in the same time), Model-free reinforcement learning method would be applied in this research. In this case, we use Q-learning with function approximation to learn the best vehicle distribution method for urban transportation.

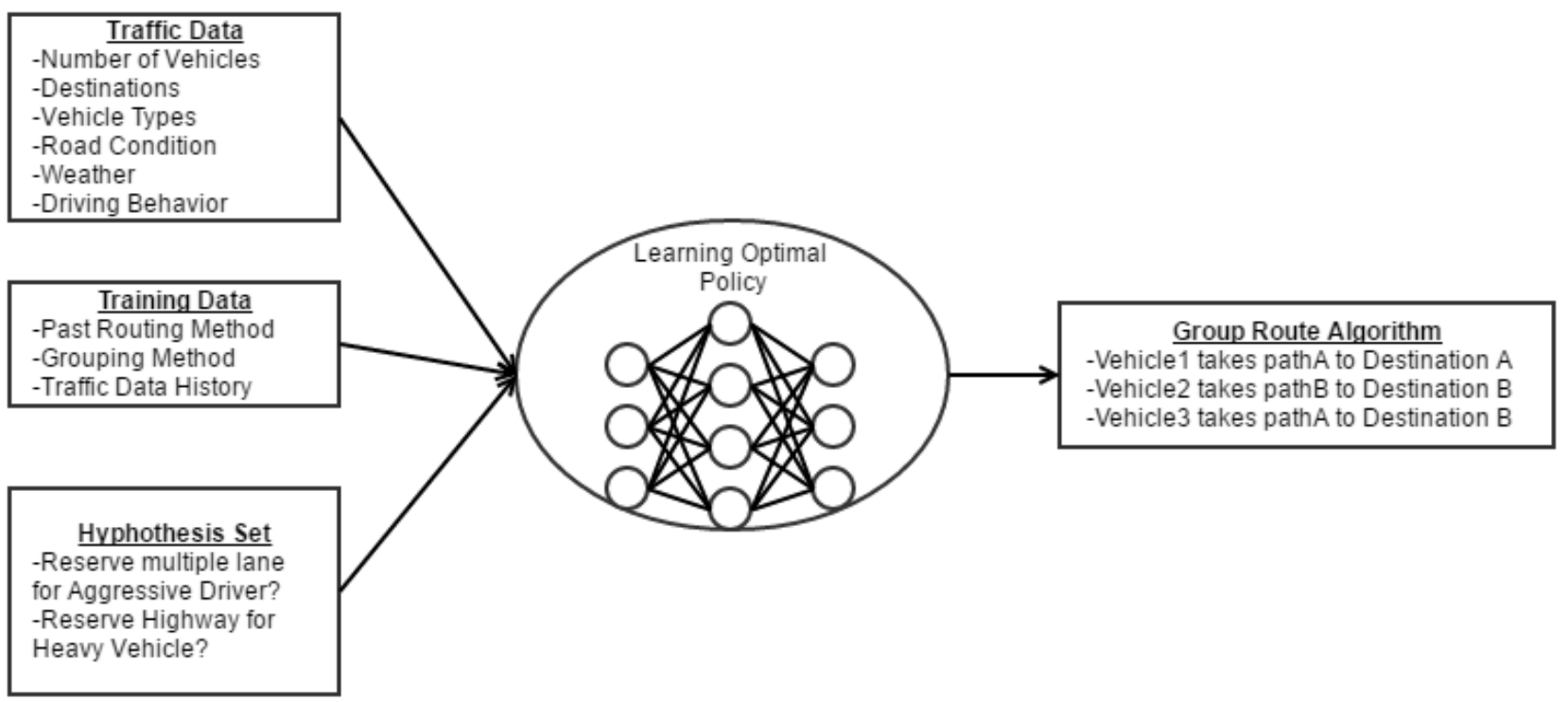

Figure 2 Learning Process of Group Route Algorithm

Q-learning algorithm is a model free reinforcement learning solution is able to find an action selection policy for MDP. Below describes how Q-learning algorithm work with general Markov Decision Process (MDP). Under a MDP with fixed transition probabilities and rewards, the Bellman equation (Equation 1) gives the optimal policy,

$$
Q(s, a)=R\left(s^{\prime}\right)+\gamma \max Q\left(s^{\prime}, a^{\prime}\right)
$$

Where $\mathrm{Q}(\mathrm{s}, \mathrm{a})$ is the immediate reward $\mathrm{R}\left(\mathrm{s}^{\prime}\right)$ for making an action plus best utility $\mathrm{Q}$ for the resulting state $\mathrm{Q}\left(\mathrm{s}^{\prime}, \mathrm{a}^{\prime}\right)$. For each state and action pair $\mathrm{Q}(\mathrm{s}, \mathrm{a})$, initialize its value to zero, then observe the current state $\mathrm{s}$ by repeating the following step:

- Pick an action and execute it

- Receive immediate reward R

- Observe new state s'

- Update Q(s, a) with the equation above.

- $\quad$ Set $\mathrm{s}=\mathrm{s}^{\prime}$

Bellman equation plays an important role in reinforcement learning, it is widely applied during policy assessment [11]. If the Q function can be correctly estimated, a better policy will become the optimal policy and lead the actions according to Equation 2.

$$
\pi(s)=\arg \max Q(s, a)
$$

With the Q-learning approach, a few key values can be extracted, including the value for the state features, action, reward and the next state features when it works together with simulator. For the details of the simulator will be discussed in the next session.

The main objective function for group route optimization is to minimize the total travel time every driver of urban transportation network. Therefore, it could lead to reduced carbon dioxide emission, and decreased gas consumption, etc. The reward of this approach is basically inherited from the objective function, which is to minimize the total travel time of the group of vehicles in the urban transportation network. This project specifically chose the travel time as the reward function of this research. Note that the travel time is not based on individual driver but the whole group of drivers on the urban transportation.

The action of this approach is rather simple which only contains path selection. With a certain level of average waiting time, the agent will choose which car to go which path for group route optimization. For example, when a car at an T-Junction, it may choose from the actions "Turn left", "Turn right" and "U-Turn".

The state features are the properties that show the situation of the traffic. In this case this project uses average waiting time of each vehicle in the traffic to determine the busy level of the urban transportation network.

Figure 2 shows the general learning process of group route algorithm. There are three major inputs in the learning process which are Traffic Data, Training Data and Hypothesis Set, and with the learning process, it will generate one output, which is the group route algorithm.

Traffic data sets reflect the real time traffic situation of the urban transportation network, which contains the total number of vehicles in the urban transport network, their current 
location, vehicle types, driving behaviour etc. The training data sets however provide a reference for algorithm to learn. It includes the past routing method, the vehicle grouping and history of past traffic data. Lastly, hypothesis sets give a set of routing theory that defined by human to allow algorithm to determine if it is valid via its learning process.

With these inputs, we can set a learning optimal policy for algorithm with neural network structure. From the learning optimal policy, the algorithm will pick the best method to reroute the traffic based on the input accordingly.

\section{PROTOTYPE}

This research used SUMO (Simulation of Urban Mobility) as the tool for simulation. SUMO is an open source, microscopic, multi modal traffic simulator. It allows the user to simulate how a specified traffic demand performs on a given road network [14].

With the map and vehicle files, SUMO is able to perform routing function to generate its route file. The default routing method will be DuaRouter, which performs dynamic user assignment (DUA) based on shortest path computation. This research will purpose another group route algorithm and demo the algorithm by using SUMO simulator, which will be discussed in the next session. DuaRouter will be the baseline of the comparison against the proposed group route algorithm.

The goal of this research is to have the group route algorithm to learn the optimal policy (which path to decide based on vehicle type and driving behavior) based on training model set. To this end, 2 features are highlighted for justifying the traffic situation.

- Average Waiting Time - SUMO provides waiting time for each car so it is possible to calculate the average waiting time to determine whether a congestion occurs.

- $\quad$ Road condition - SUMO provides details information of occupancy, stored travel time in each lane, which could be used to determine the busy level of a lane.

SUMO also provides details parameters of each vehicles. Therefore, this research could easily calculate the average travel time of every vehicle and uses it to decide if a reward or punishment should be taken while doing reinforcement learning.

SUMO allows user to set the specification of the vehicle features, which are corresponding to different vehicle type and driving behavior.

With the features above, SUMO is an excellent choice for this research with its ability to perform an optimized traffic distribution method based on vehicle type or driver behavior in order to maximize the capacity of the urban transportation, and also update the vehicle's route when congestion or accident occurs. It also supports TraCI, a Python API that allows a user to get information from a traffic simulation and modify the simulation as it runs in response to this information.

\section{EXPERIMENTS}

We setup a simple experiment to check how much the traffic will be improved by redirecting the vehicles based on their similarities to reduce the dependency. In this experiment, we set a transport network which contains a square roadway as shown in Figure 3.

We defined 3 different vehicle types (fast car, normal car and truck) in SUMO rou.xml file as shown below:

$<$ vType accel="3.0" decel="6.0" id="fastcar" length="5.0" minGap $=" 2.5$ " maxSpeed="50.0" sigma $=" 0.5$ " color="red" $>>$

$<$ vType accel="2.0" decel="5.0" id="normalcar" length="5.0" maxSpeed $=" 40.0$ " sigma $=$ "0.5" color="yellow" $>$

$<$ vType accel="1.0" decel="4.0" id="truck" length="8.0" maxSpeed="20.0" sigma="0.5" color="green"/>

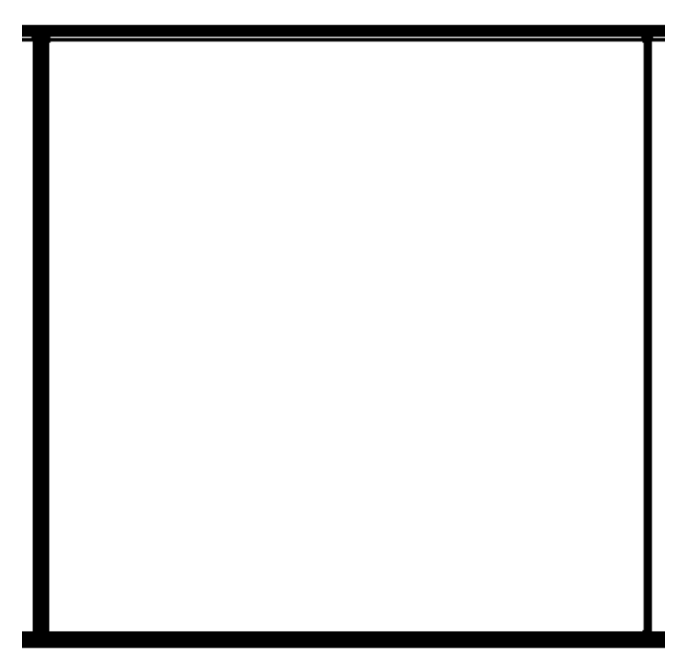

Figure 3 Network for experience

From Figure 4 we could see in experiment A, we first put total 120 normal cars, 60 fast cars and 20 trucks in our simulation network, with the trips from top left point to bottom right point. Those vehicles were equally assigned to 2 different routes (one route contain 60 normal cars, 30 fast cars and 10 trucks), route A is from top left point to bottom left point then to bottom right point, and route B is from top left point to top right point then to bottom right point and run the simulation

After that in experiment B we assigned the route of vehicles based on the vehicles types. We put 60 fast cars and 50 normal cars in route A, 70 normal cars and 20 trucks in route $B$ then run the simulation again.

From 2 simulations, experiment 2 that route with similar group have much lesser simulation time than experiment 1 . It is because the vehicles could travel to their destination with less dependency.

This experiments shows that by managing the traffic based on the similarities of the vehicles could potentially reduce the total travel time of the urban transportation network.

\begin{tabular}{|l|l|l|l|}
\hline Route 1 & Route 2 & $\begin{array}{c}\text { Simulation } \\
\text { Time }\end{array}$ \\
\hline
\end{tabular}




\begin{tabular}{|l|l|l|l|}
\hline Exp A & $\begin{array}{l}60 \text { normal cars, } \\
30 \text { fast car, } \\
10 \text { trucks }\end{array}$ & $\begin{array}{l}60 \text { normal cars, } \\
30 \text { fast car, } \\
10 \text { trucks }\end{array}$ & 325 \\
\hline Exp B & $\begin{array}{l}50 \text { normal cars, } \\
60 \text { fast cars }\end{array}$ & $\begin{array}{l}70 \text { normal cars, } \\
20 \text { trucks }\end{array}$ & 262 \\
\hline
\end{tabular}

Figure 4 Simulaiton Result

\section{CONCLUSION AND FUTURE WORK}

This paper studied a new method of manage the urban traffic by routing group of vehicles to their destination based on the similarities of vehicles. We believe this method could possibly reduce the dependency of the roadway and hence reduce the traffic congestion and bring the best benefit to urban perspective.

In summary, we discussed the design of the general flow of group routing method and how it is going to help with the urban traffic management. We also studied the learning method of this approach and how it is going to work with the prototype.

The next phase of this research will be the evaluation of the group route optimization with reinforcement learning method and the development of the prototype. We will setup the training set for the optimal policy of group routing method and research group routing method associated with sustainability and resilience.

\section{REFERENCE}

[1] Gilbert Laporte. The Vehicle Routing Problem: An Overview of Exact and Approximate Algorithms. 1991

[2] E. Dijkstra: ' A Note on Two Problems in Connexion with Graphs', Numerische Mathematik, Vol. 1, pp. 269-271. 1959.

[3] Dorigo, M., Maniezzo, V., \& Colorni, A. Ant System: Optimization by a colony of cooperating agents. IEEE Transactions on Systems, Man, and Cybernetics-Part B, 26(1), 29-41. 1996

[4] Nahar, S. A.A. and Hashim, F.H. 'Modelling and analysis of an efficient traffic network using ant colony optimization algorithm', in Third International Conference on Computational Intelligence, Communication Systems and Networks (CICSyN), pp.32-36, IEEE. 2011

[5] Kammoun, H.M., Kallel, I., Alimi, A.M. and Casillas, J. 'An adaptive vehicle guidance system instigated from ant colony behavior', IEEE International Conference on Systems Man and Cybernetics (SMC), pp.29482955, IEEE.

[6] Zong, X., Xiong, S., Fang, Z. and Li, Q. (2010) 'Multi-ant colony system for evacuation routing problem with mixed traffic flow', in IEEE Congress on Evolutionary Computation (CEC), pp.1-6, IEEE. 2010.

[7] Cong, Z., DeSchutter, B. and Babuska, R. 'A new ant colony routing approach with a trade-off between system and user optimum', in 14th International IEEE Conference on Intelligent Transportation Systems (ITSC), pp.1369-1374, IEEE. 2011.

[8] Kponyo, J.J., Kuang, Y. and Li, Z. 'Real time status collection and dynamic vehicular traffic control using ant colony optimization. International Conference on Computational Problem-Solving (ICCP), pp.69-72.2012.

[9] Richard S. Sutton, Andrew G. Barto. Reinforcement Learning: An Introduction. 2012

[10] Kapileswar Nellore and Gerhard P. Hancke. A Survey on Urban Traffic Management System Using Wireless Sensor Networks. January 2016.

[11] Kappen, Hilbert J, Gomez, Vicenc, , and Opper, Manfred. ' Optimal control as a graphical model inference problem. Machine learning, 87(2):159-182, 2012.

[12] Chao, K.H.; Chen, P. An intelligent traffic flow control system based on radio frequency identification and wireless sensor networks. Int. J. Distrib. Sens. Netw. 2014.

[13] R.Bruno, M.Nurchis. Robust and efficient data collection schemes for

vehicular multimedia sensor networks. In Proceedings of the IEEE 14th
International Symposium and Workshops on World of Wireless, Mobile and Multimedia Networks (WoWMoM), Madrid, Spain. 2013.

[14] Michael Behrisch, Laura Bieker, Jakob Erdmann, Daniel Krajzewicz. SUMO - Simulation of Urban Mobility. The Third International Conference on Advances in System Simulation. 2011.

[15] Gilbert Laporte. The Vehicle Routing Problem: An Overview of Exact and Approximate Algorithms. 1991 